\title{
Family Based Rehabilitation: Pressing Priority for Rehabilitative Needs of Chronic Patients in COVID Times
}

\author{
Rishabh Sharan ${ }^{1}$, Anu Arora ${ }^{2}$ \\ ${ }^{1}$ PhD. Scholar (CIDR, D.Y. Patil University), Assistant Professor, Department of Neuro Physiotherapy, School of \\ Physiotherapy, D.Y. Patil Deemed to be University, Navi Mumbai, 400706, ${ }^{2}$ Associate Professor, Department of \\ Physiotherapy in Community Health, School of Physiotherapy, D. Y. Patil Deemed to be University, Navi Mumbai
}

\begin{abstract}
The health care sector is currently facing twin burden, the impact of coexisting diseases as well as the pandemic of corona virus. The importance of commencing Neuro rehabilitation as early as possible is now well appreciated and accepted with adequate supporting evidence. However, it is also a fact that proper \& prompt rehabilitation services are often deficient in majority of developing countries. With the everincreasing burden of those affected with the highly infectious disease COVID-19, venturing to Physiotherapy OPD for routine rehab care is not at all safe for the already vulnerable clients.

At this point of time, family members can function as an extension of health care system by co-ordinating and contributing by participation, in the rehabilitation as directed by the therapist via telemedicine. Remote delivery of Physiotherapy through guided family-based approach is an ideal model to support on-going Physiotherapist's services during this period, especially in the most vulnerable clients.
\end{abstract}

Keywords: COVID 19, Family Based Rehabilitation, Tele-medicine, Neurological Disorders.

\section{Introduction}

The health care sector is currently facing twin burden the impact of coexisting diseases as well as the pandemic of corona virus. To add more gravity to the already grim situation is the fact that $\mathrm{WHO}$ has documented a massive burden associated with neurological disorders and has supplanted that neurological services and resources are disproportionately scarce, especially in developing \& low-income countries. ${ }^{1}$

The importance of commencing Neuro rehabilitation as early as possible is now well appreciated and accepted with adequate supporting evidence. ${ }^{2}$ However, it is also a fact that proper \& prompt rehabilitation services are often deficient in majority of developing countries. Policy makers and health care providers may be under prepared to cope up with the predicted rise in the prevalence of neurological and other chronic disorders and the disability resulting from the extension of life expectancy and ageing of populations globally. ${ }^{1}$ The situation is further made grim by the fact that neurological dysfunction is linked to cardiopulmonary deficits due to impaired mobility and functioning, aging leading to deprived cardiopulmonary endurance and deconditioning. ${ }^{3}$ All these factors make early initiation of therapy and thereby movement imminent.

With the ever-increasing burden of those affected with the highly infectious disease COVID-19, how safe is it for everyone to step out? The question might be rhetoric, but the problem deserves an appropriate solution.

When faced with such a rampant contagion scenario, how wise and humane is it to expect our neurologically affected patients to get treated on an OPD basis, amidst of all this? Because venturing to Physiotherapy OPD for routine rehab care is like asking the already vulnerable clients, who already are going through turmoil in their internal system, to further risk the external ailments in their stride.

With much of the country paralysed by fear and frustration, the circumstances could be potentially damaging to health as staying home with little to no social interactions not only would halt the recovery but also has proven to increase the risk of developing mental health conditions such as depression and anxiety. 
"The fallout from the pandemic - job losses, prolonged stress and a deterioration of mental health will be felt by families for years to come," said UNICEF Chief of Early Childhood Development Dr. Pia Rebello Britto. This is not an unknown fact that number of cases are staggeringly increasing, good immunity is required to fight the pandemic. This is only possible with an obvious social distancing, in addition to which, overall fitness \& nutrition also play a crucial role. ${ }^{4}$ Community transmission as an impending risk, travelling up to the physiotherapist itself can summon the virus. Queuing up at hospitals or clinics may expose people to sit or stand a chance for others to get infected. Older adults are at a significantly increased risk of severe disease following infection from COVID-19.

Patients with neurological deficits as important as they need rehabilitation ${ }^{2}$ can't ignore the fact that their health condition taking a toll on their immune system. Getting immunity jeopardized at this peak of hour by discontinuing rehabilitation at physiotherapy OPD/ clinic \& resting at home is no wise man's decision! The possible steps to ensure safety at both ends \& mitigate the virus and create a sterile environment for every patient in the OPD/clinic are economically exhausting. If we can change our shopping pattern during the pandemic alarming situation, why cannot we change our treatment patterns? This calls for the rising need for family-based rehabilitation (FBR).

\section{Discussion}

Family being an essential key in this integrated process $^{5}$ necessitates more and more therapists to come up with this structured approach, so that this helps us reach to masses and downsize the suffering through neurological disorders to a great extent without imperilling our patients. Continuity being an important part of process of Neuro-rehabilitation, effective strategies can be implemented to integrate family into achieving the patient outcomes as far as possible.

At this point of time, family members can function as an extension of health care system by co-ordinating and wholeheartedly participating in the exercise regime as directed by the therapist via telemedicine. This era of time where technology meets health is a boon to most of us taking care of financial cost as it poses both a real and perceived barrier to the application and adoption of Tele rehab. ${ }^{6}$

Delving on the different forms of rehabilitation family based tele-rehabilitation has the potential to prove to be the most effective for intense long-term rehabilitation of individuals with neurological ailments. This treatment model can be inexpensive but effective and can be encouraged to practice in rehabilitating various neurological disorders.

Family-based in-home treatment can effectively meet the needs of client as well as the care givers struggling with the dual challenges preventing the infection as well as providing optimum therapeutic care.

Hence in current uncertain times demanding a need for social distancing, implementation of Family Based Rehabilitation Models is very important. Remote delivery of Physiotherapy through guided familybased approach is an ideal model to support on-going Physiotherapist's services during this period, especially the most vulnerable of the clients.

For optimum efficiency of this regime, some pertinent questions need to be answered before implementation of a Family Based Rehabilitation:

- Is Family Based Rehabilitation appropriate for this client?

- Does the therapist have the skills and training to provide remote physiotherapy to the patients?

- Are the techniques of Family Based Rehabilitation rooted in an evidence-based approach?

- Can available Assistive Technology be incorporated into rehab regimes to make them more efficient?

- Can some form of support in form of telerehabilitation be included for remote proctoring of the management?

- How the immediate and long-term goals for management be stipulated?

- Is the imparted rehabilitation model in sync with the expectations and calibre of the client and the family members?

\section{Conclusion}

Keeping the families safe and together, the FBR approach places emphasis on the needs of the client as well as the comfort and safety of client as well as the care givers. However, because these programs are relatively new, there is an imminent need to take on diligent and robust research to answer the some of the above-mentioned queries and thereby launch FBR for 
management of subjects who require long term care. Not only would it lay down the road map for rehab care plans in case of contingencies but also serve to put forth an ideal adjunct rehab care plan for subjects with varying needs even in times of peace. With appropriate Program design, implementation FBR seems to be a promising model for the future of therapeutic care for chronic patients.

Ethical Clearance: Was not obtained (the manuscript is written as a brief theoretical article category for a novel concept).

Source of Funding: Self.

\section{Conflict of Interest: Nil.}

\section{References}

1. GBD 2016 Neurology Collaborators. Global, regional, and national burden of neurological disorders, 1990-2016: a systematic analysis for the Global Burden of Disease Study 2016. Lancet Neurol. 2019 May;18(5):459-480. doi: 10.1016/ S1474-4422(18)30499-X.
2. Kleim JA, Jones TA. Principles of experiencedependent neural plasticity: implications for rehabilitation after brain damage. J Speech Lang Hear Res. 2008 Feb;51(1):S225-39. doi: 10.1044/1092-4388(2008/018).

3. Polkey MI, Lyall RA, Moxham J, Leigh PN. Respiratory aspects of neurological disease. J NeurolNeurosurg Psychiatry. 1999 Jan;66(1):5-15. doi: 10.1136/jnnp.66.1.5.

4. Tay MZ, Poh CM, Rénia L, MacAry PA, Ng LFP. The trinity of COVID-19: immunity, inflammation and intervention. Nat Rev Immunol. 2020 Jun;20(6):363-374. doi: 10.1038/s41577-0200311-8.

5. Norup A. Family matters in neurorehabilitation: why, when, who, and how? Revistalberoamericana de Neuropsicología. 2018;1(1):17-31.

6. Sarfo FS, Ulasavets U, Opare-Sem OK, Ovbiagele B. Tele-Rehabilitation after Stroke: An Updated Systematic Review of the Literature. J Stroke Cerebrovasc Dis. 2018 Sep;27(9):2306-2318. doi: 10.1016/j.jstrokecerebrovasdis.2018.05.013. 